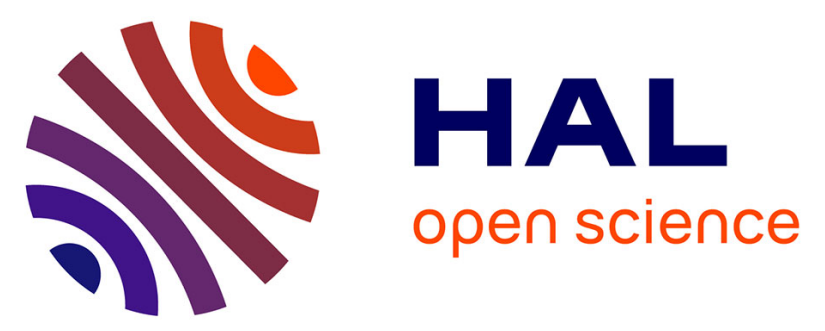

\title{
Magnetic reorientation and thermal stability in MnAs/GaAs (100) micro patterns driven by size effects
} M. Tortarolo, F. Fernandez Baldis, M. Sirena, L. B. Steren, J. Milano, Victor H. Etgens, Mahmoud Eddrief, G. Faini

\section{- To cite this version:}

M. Tortarolo, F. Fernandez Baldis, M. Sirena, L. B. Steren, J. Milano, et al.. Magnetic reorientation and thermal stability in MnAs/GaAs (100) micro patterns driven by size effects. Journal of Applied Physics, 2012, 112 (1), pp.013915. 10.1063/1.4733698 . hal-01240932

\section{HAL Id: hal-01240932 \\ https://hal.science/hal-01240932}

Submitted on 22 Apr 2020

HAL is a multi-disciplinary open access archive for the deposit and dissemination of scientific research documents, whether they are published or not. The documents may come from teaching and research institutions in France or abroad, or from public or private research centers.
L'archive ouverte pluridisciplinaire HAL, est destinée au dépôt et à la diffusion de documents scientifiques de niveau recherche, publiés ou non, émanant des établissements d'enseignement et de recherche français ou étrangers, des laboratoires publics ou privés. 


\title{
Magnetic reorientation and thermal stability in MnAs/GaAs (100) micro patterns driven by size effects
}

\author{
M. Tortarolo, ${ }^{1}$ F. Fernandez Baldis, ${ }^{2,3}$ M. Sirena, ${ }^{2,3}$ L. B. Steren, ${ }^{3,4}$ J. Milano, ${ }^{2,3}$ \\ V. H. Etgens, ${ }^{1,5}$ M. Eddrief, ${ }^{1}$ and G. Faini ${ }^{6}$ \\ ${ }_{1}^{1}$ Institut des NanoSciences de Paris, UPMC, CNRS-UMR 7588, 75015 Paris, France \\ ${ }^{2}$ Centro Atómico Bariloche CNEA, 8400 S. C. de Bariloche, Argentina \\ ${ }^{3}$ Consejo Nacional de Investigaciones Científicas y Técnicas, C1033AAJ Buenos Aires, Argentina \\ ${ }^{4}$ Centro Atómico Constituyentes CNEA, 1650 San Martín, Argentina \\ ${ }^{5}$ Fédération Lavoisier Franklin, UVSQ, 78035 Versailles Cedex, France \\ ${ }^{6}$ Phynano Team, CNRS, Laboratoire de Photonique et de Nanostructures, 91960 Marcoussis, France
}

(Received 24 February 2012; accepted 5 June 2012; published online 11 July 2012)

\begin{abstract}
Size effects and their consequences in the thermal stability of the magnetization of the micro-sized $\mathrm{MnAs} / \mathrm{GaAs}(100)$ ribbons were studied by magnetic force microscopy. We found out that the orientation in which size is reduced plays a key role in the magnetic configuration and thermal stability of the micro-sized patterns. On the one hand, when reducing the size in the [0001] $\alpha$-MnAs direction, the system shows an improvement in the thermal stability of the remanent magnetization. On the other hand, when the size is reduced in the [11-20] $\alpha$-MnAs direction, the system goes through a magnetic reconfiguration from in-plane magnetized to out-of-plane magnetized, which also improves the thermal stability. (C) 2012 American Institute of Physics. [http://dx.doi.org/10.1063/1.4733698]
\end{abstract}

\section{INTRODUCTION}

Previous publications ${ }^{1-3}$ evidenced the role played by substrate-induced strains on self-organized MnAs/GaAs (100) patterns. Particularly, much attention is being paid to the strain effects arising from the crystalline misfit at interfaces of thin films. These effects are gathered in a very active field called strain-engineering whose aim is to reach the required properties by handling film strains. ${ }^{4,5}$ In magnetic media, any alteration of the strain distribution may have strong consequences on the magnetic properties of the devices. ${ }^{6,7}$ These properties could be tuned by controlling either the strains or the temperature. , $^{8}$

MnAs can be grown epitaxially on different GaAs orientations by molecular beam epitaxy (MBE), which makes it promising for hybrid metal/semiconductor spintronics. ${ }^{10}$ The physical properties of thin films are different from the bulk ones. ${ }^{11-13}$ On the one hand, bulk MnAs exhibits a first-order phase transition slightly above $40^{\circ} \mathrm{C}$, from a hexagonal ferromagnetic $(\alpha)$ to an orthorhombic paramagnetic one $(\beta)$. On the other hand, in MnAs films grown on GaAs, the $\alpha$ and $\beta$ phases coexist in a wide range of temperatures below Tc, depending on the growth conditions. ${ }^{14}$ Both phases form stripes that lay along the c-axis and are periodically arranged all over the samples in MnAs films grown onto (100) GaAs. ${ }^{15}$ The particular arrangement of magneto-elastic domains has been modeled in Ref. 16 and explained in terms of the effect of substrateinduced strains effect on the magneto-structural phase transition. Coelho and coworkers ${ }^{9}$ studied the temperature evolution of the magnetic domains up to the Curie temperature, observing a change of the magnetic domains structure with the progressive narrowing of the $\alpha$-stripes. In this article, we report on the low-temperature magnetic domain structure of MnAs micro and nanoribbons and the thermal stability of the remanent state in these structures, studied by imaging the magnetic contrast of the samples as a function of temperature.

\section{EXPERIMENTAL}

A $150 \mathrm{~nm}$ thick MnAs film was grown by MBE on a GaAs(100) substrate as described elsewhere. ${ }^{17}$ The film grown in As rich conditions has the following orientations ${ }^{18,19}: \operatorname{MnAs}[1100] / / \operatorname{GaAs}(100)$ and $\operatorname{MnAs}[0001] / /$ GaAs(1-10). A pattern of ribbons of different sizes and orientations was transferred to the film by e-beam lithography followed by Ar ion milling. The ribbons widths, $w$, were chosen in reference to the period of the $\alpha / \beta$ stripes array in the as-cast MnAs film $\left(\lambda_{\mathrm{e}} \sim 4.8 . t=0.72 \mu \mathrm{m}\right.$ for the present sample $\left.^{20}\right)$. We fabricated $2 \mu \mathrm{m} \leq w \leq 5 \mu$ m width ribbons larger than $\lambda_{\mathrm{e}}$; others with $0.5 \mu \mathrm{m} \leq w \leq 1 \mu \mathrm{m}$ comparable to $\lambda_{\mathrm{e}}$; and $0.1 \mu \mathrm{m} \leq w \leq 0.2 \mu \mathrm{m}$ ribbons smaller than $\lambda_{\mathrm{e}}$. The samples were carefully aligned in order to confine the ribbons along the MnAs [0001] direction (S1) and the [11-20] direction (S2) (see Fig. 1). The images showed in this work were taken at several temperatures between $11^{\circ} \mathrm{C}$ and $45^{\circ} \mathrm{C}$ in the remanent state, after magnetizing the sample at room temperature in a magnetic field of $1 \mathrm{~T}$ applied along the easy-axis of magnetization ([11-20]). ${ }^{17,21}$

\section{RESULTS AND DISCUSSION}

As reference, the temperature dependence of the magnetic domain structure of a MnAs thin film was measured. At room temperature, the remanent state of the film consists of in-plane magnetized single-domains arranged in the typical $\alpha / \beta$ stripes array of MnAs/GaAs(100). ${ }^{15}$ The magnetization 


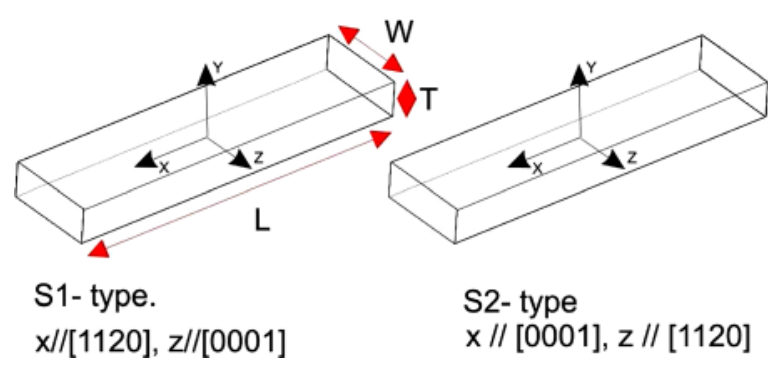

FIG. 1. Ribbons oriented parallel (S1) and perpendicular (S2) to the crystallographic $c$-axis; the [11-20] direction of the $\alpha$-MnAs lattice.

of each $\alpha$-stripe lays along the [11-20] easy-axis. The period measured for these stripes was $\lambda_{\mathrm{m}} \sim 0.74 \mu \mathrm{m}$, in excellent agreement with the estimated value, $\lambda_{\mathrm{e}} \cdot{ }^{22}$ When the temperature is increased some of the domains reverse their magnetization and the dominant domain structure in the $\alpha$ phase becomes a meander-like in-plane magnetized structure referred as type I in Ref. 23. At higher temperatures, close to $\mathrm{T}_{\mathrm{c}}$, the $\alpha$ stripes turn into ferromagnetic islands embedded in the $\beta$ matrix and the magnetic contrast progressively vanishes.

Room-temperature magnetic force microscopy (MFM) measurements, reported in Ref. 24, showed the existence of notable anisotropic size effects on the array of magnetostructural domains in ribbons. The lateral confinement of MnAs thin films leads to notably effects on the thermal stability of the magnetic phase and domain structure. The domain configuration at $11^{\circ} \mathrm{C}$ shows already a clear dependence on the ribbons size and orientation (Figs. 2 and 4).

The magnetic domain structure of the $\mathrm{S} 1$ widest ribbons resemble that of the thin films. A smooth corrugation of the magnetic signal is observed at the surface of these samples at $11^{\circ} \mathrm{C}$ (Figs. 2(a) and 2(c)). The magnetic domain structure behaves as the unconfined films, evolving from a saturated state to a meander-like structure at higher temperatures (Figs. 2(b) and 2(d)).

The periodicity of the $\alpha / \beta$ array, $\lambda$ decreases with the ribbons width as shown in Fig. 3. The confinement of the
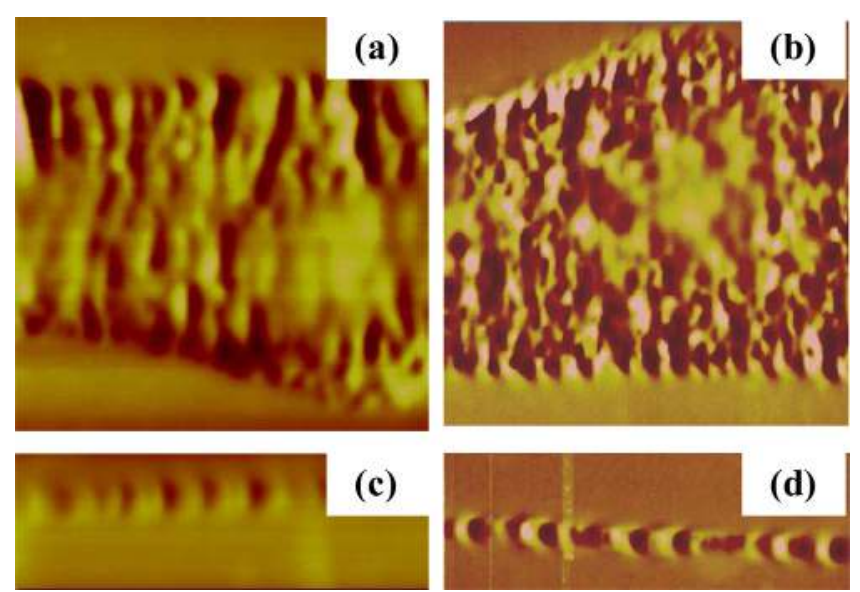

FIG. 2. MFM images of MnAs/GaAs(001) ribbons confined in the [0001] $\alpha$-MnAs direction and measured at different temperatures ( $\mathrm{S} 1$ series): (a) $w=5 \mu \mathrm{m}, \mathrm{T}=11^{\circ} \mathrm{C}$; (b) $w=5 \mu \mathrm{m}, \mathrm{T}=43^{\circ} \mathrm{C}$; (c) $w=0.2 \mu \mathrm{m}, \mathrm{T}=11^{\circ} \mathrm{C}$; and (d) $w=0.2 \mu \mathrm{m}, \mathrm{T}=43^{\circ} \mathrm{C}$, respectively.

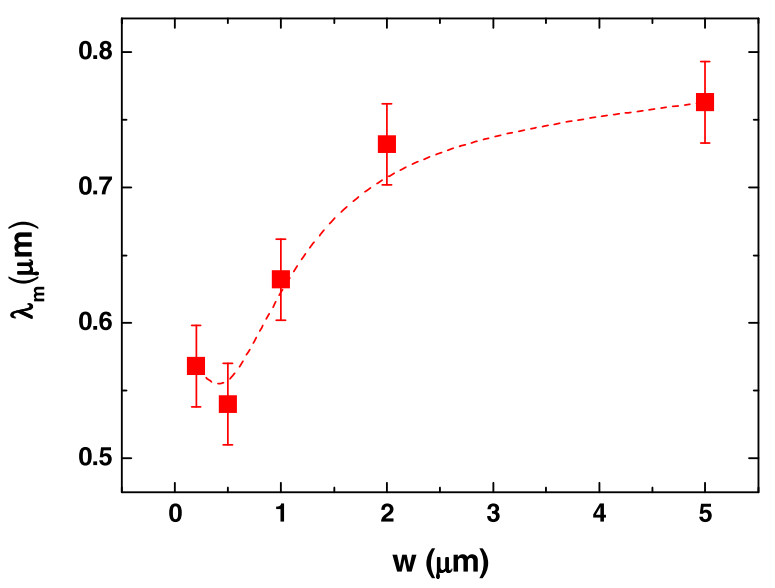

FIG. 3. Stripes period, $\lambda_{\mathrm{m}}$ vs. ribbons width $w$. Data extracted from MFM images taken at $17^{\circ} \mathrm{C}$ (Fig. 2).

film to a thin wire does change the elastic energy of the system, noticed as a size effect on the period of the elastic domains arrangement. Strains induced at the ribbons free surface normal to the [0001] axis would affect the Young modulus ${ }^{25,26}$ in the ribbons axial direction and consequently change the period $\lambda$. The model proposed by Kaganer et al. ${ }^{1}$ neglects z-components of the strain tensor and our results indicate that it must be taken into account to explain the nanostructures behaviour.

The $\alpha$ stripes are still observed at the higher temperatures in the narrowest ribbons, showing a higher stability of the magnetic domains in the nanoribbons. In these cases, the ferromagnetic phase is uniformly magnetized along the [11-20] in single-domain states that have a nearly square cross-section in the $\mathrm{x}-\mathrm{z}$ plane with a side, $w \sim 0.3 \mu \mathrm{m}$.

The MFM images show that there is a slight decrease of the average remanence with increasing temperature, i.e.,: some S-D reverse but $M r / M s$ along the [11-20] direction is still $\sim 1$ till high temperatures. A loss of magnetic contrast is also observed as the temperature is increased.

The magnetic domain structure of the S2-ribbons is completely different from the one observed in the previous series of samples. The S2 samples show a passage from an uniformly magnetized state to multidomain states, regular and comb-like shape, respectively, as the width of the ribbons decreases from a few microns to a few tenths microns (Fig. 4). The $5 \mu \mathrm{m} \mathrm{S2-ribbon} \mathrm{is} \mathrm{single-domain} \mathrm{at} 11^{\circ} \mathrm{C}$ while the remanent magnetization of the other ribbons splits into a multi-domain structure. The magnetic domain structures of the narrower bars depend markedly on their width, $w$. In $2 \mu \mathrm{m}$ and $1 \mu \mathrm{m}$-thick bars, the magnetization points along the [11-20] easy-axis in domains anti-parallel aligned along the [0001] direction (see sketch in Fig. 4(c)). The domains are extended along the total width of the bars and their average length $D$ along the c-axis decreases with the width of the samples from $D=0.9 \mu \mathrm{m}$ for $w=2 \mu \mathrm{m}$ to $D=0.45 \mu \mathrm{m}$ for $w=1 \mu \mathrm{m}$. The domain walls are not observed, so they are assumed to be extremely thin.

The narrower bars show a comblike domain structure ${ }^{27}$ with domains of irregular shape and elongated along the [0001] direction (Fig. 4(e)). The complex comblike structure 

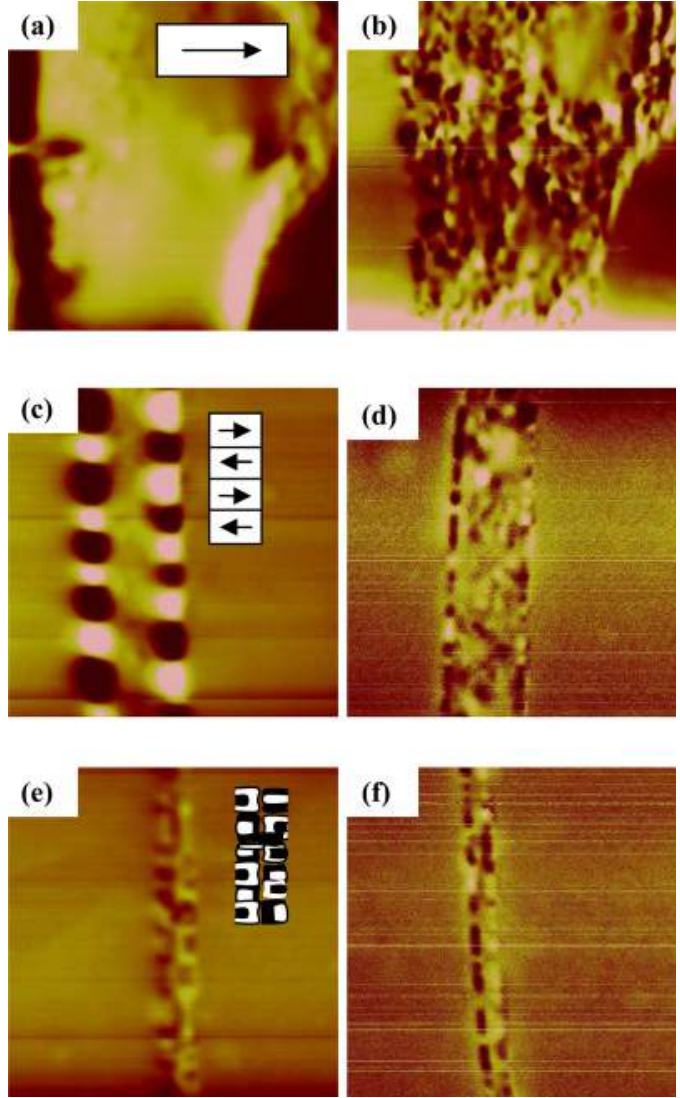

FIG. 4. MFM images of $\mathrm{MnAs} / \mathrm{GaAs}(100)$ ribbons confined along the [1120] axis measured at differente temperatures (S2 series): (a) $w=5 \mu \mathrm{m}$, $\mathrm{T}=11^{\circ} \mathrm{C}$; (b) $w=5 \mu \mathrm{m}, \mathrm{T}=43^{\circ} \mathrm{C}$; (c) $w=2 \mu \mathrm{m} \mathrm{T}=11^{\circ} \mathrm{C}$; (d) $w=2 \mu \mathrm{m}$ $\mathrm{T}=43^{\circ} \mathrm{C}$; (e) $w=0.5 \mu \mathrm{m} \mathrm{T}=11^{\circ} \mathrm{C}$; and (f) $w=0.5 \mu \mathrm{m} \mathrm{T}=11^{\circ} \mathrm{C}$.

had been already observed in some regions of MnAs thin films and simulated by R. Engel-Herbert and coworkers. ${ }^{28}$ These domains appear to be the more stable structures for sub-micrometric MnAs S2-ribbons and have a 3D texture.

The low-temperature domain structure of the larger bars can be described by analyzing the energy balance between demagnetizing and domain wall terms. The magnetostatic energy density for a uniformly magnetized state depends linearly on the demagnetizing factor, $N_{d}$. The value of $N_{d}$ for a rectangular prism in the limit of infinite length along one direction (in our case $\mathrm{x} / /[0001]$ ) has been derived by Aharoni. ${ }^{29}$ For a system magnetized along $z$, i.e., [11-20], the demagnetization factor in the z-direction is given by

$$
N_{d, z}=\frac{1-p^{2}}{2 p} \cdot \ln \left(1+p^{2}\right)+p \cdot \ln (p)+2 \cdot \arctan g\left(\frac{1}{p}\right),
$$

with $p=w / t$. The demagnetizing factor in the $x=$ [0001] direction is neglected and thus the $\mathrm{N}_{\mathrm{d}, \mathrm{y}}=1-\mathrm{N}_{\mathrm{d}, \mathrm{z}}$. The value of $N_{d z}$ increases with decreasing $p$, being almost equal to 0.5 for our narrower samples. Below a critical ribbon width, the single-domain state energy at low temperatures is larger than that of the multidomain state and the transition between both configurations occurs. In the multidomain state, the magnetic domains of thickness $D$ point along the anisotropic easyaxis. The size of the domains depends on the wall energy per unit area $\gamma$, the width of the ribbons $w$, and the saturation magnetization $M_{S}$. A rough estimation of $D$ for a magnetic slab is given by ${ }^{30}$

$$
D=\sqrt{\frac{\gamma w}{0.85 M_{s}^{2}}} \approx 0.25 \mu \mathrm{m},
$$

taking $\gamma=0.011 \mathrm{~J} / \mathrm{m}^{2}$ (Ref. 27) and $\mathrm{M}_{\mathrm{s}}=4 \times 10^{5} \mathrm{~A} / \mathrm{m}^{17}$

The limit of the domain model is the case $N_{d y}=N_{d z}$ $\sim 0.5$ and is reached for our narrower bars where in fact a different domain structure is observed (see above). No evidences of $\beta$ phase are found at the surface of any of the S2-ribbon at $11{ }^{\circ} \mathrm{C}$. As the temperature is increased, the magnetic domain structure in the larger ribbon evolves in the same way as that of the film (Fig. $4(\mathrm{~b})$ ). At $17^{\circ} \mathrm{C}$, these ribbons show the typical corrugation of the $\alpha / \beta$ stripes arrays. The mapping of surface stray fields indicate that at surface, the fraction of magnetic moments pointing into and out of the plane of the ribbons increases with temperature in the narrower ribbons. Coelho et al..$^{9}$ showed through a simple calculation that out-of-plane domains are energetically favorable than in-plane domains at high temperatures, when the $p=w_{\alpha} / t$ ratio of the $\alpha$ stripes is smaller than 2.6. Our results indicate that in spite of the fact that magnetization tends to orientate out-of-plane due to the progressive increase of the $N_{z} / N_{y}$ ratio from 0 to 1 , the structure of the magnetic domains is not so simple as the one proposed in Ref. 9 but have a 3D magnetic texture. ${ }^{27}$ In fact, our case is rather different from that of Coelho's: we have not found $\alpha$ and $\beta$ stripes in ribbons with $w \leq 1 \mu \mathrm{m}$ when the temperature of the samples is increased above $11^{\circ} \mathrm{C}$ (Figs. 4(d) and 4(f)). We assumed that as the ribbons widths are comparable to the magneto-structural period, there is a rearrangement of the $\alpha$ and $\beta$ phases in a core-shell structure. The elastic energy in these cases favours a sandwich geometry where the $\alpha$ phase remains on the surface of the ribbon while the $\beta$ one is underneath, at the GaAs interface. ${ }^{24}$ We analyze the magnetic behaviour of MnAs ribbons as belonging from single ferromagnetic slabs of effective thickness $t^{*}$, being $t^{*} \leq t$. The easy-axis for magnetization in these slabs is dominated by the shape anisotropy over the magnetic anisotropy of MnAs.

At the highest temperatures, the S2-samples clearly still show strong magnetized regions pinned at the edges of the ribbons while they present a much smaller magnetic contrast in their center. The smallest S2 ribbon $(w=300 \mathrm{~nm})$ still shows the characteristic magnetic contrast of an OP magnetized system. In this case, the magnetic configuration is stable up to higher temperatures than the non-structured film because opposite magnetized elements stabilize each other by dipolar interactions.

\section{CONCLUSION}

In summary, we have revealed that the confinement of MnAs ribbons down to the sub-micrometric scale along the [0001] serves to stabilized the low-temperature ferromagnetic phase up to higher temperature than in thin films. These samples exhibit a more stable configuration of the stripes 
array as a function of temperature, compared to the continuous films. On the other hand, we show that confinement of MnAs ribbons to the microscale has strong effects on the domain structure of the samples. By nanostructuring MnAs films, we succeeded in isolating different types of magnetic domains, energetically favorable for particular sizes of ribbons. Otherwise, a mixture of these domains would be observed in continuous films. As the ribbons width of samples oriented along the [0001] direction is reduced, a change from a uniformly magnetized state into a multidomain state is observed. Two clearly different multidomain states can be identified depending on the size of the ribbons: rectangular and comblike ones. Moreover, as the temperature increases the comb-like structure progressively evolves to an assembly of out-of-plane magnetized domains. The results are explained in terms of changes of the magnetostatic energy with the aspect ratio of the ribbons and also intimately linked to the tensions induced by the substrate. Our results highlight the critical role of the anisotropic size effects in the system when it comes to the magnetic behaviour and thermal stability of the magneto-structural properties of MnAs/GaAs(100) micro-sized patterns.

\section{ACKNOWLEDGMENTS}

We acknowledge the partial financial support from PICT 33304 and CONICET PIP 1333. This work has been done within the LIFAN collaboration. We also acknowledge H. Pastoriza for the use of the microfabrication facilities and Mr. J. Perez and Mr. R. Benavidez for the technical support. M.T. wish to thank L. Pietrasanta and C. von Bilderling for the aid at the CMA FCEyN, UBA.

${ }^{1}$ V. M. Kaganer, B. Jenichen, F. Schippan, W. Braun, L. Daweritz, and K. H. Ploog, Phys. Rev. B 66, 045305 (2002).

${ }^{2}$ Y. Takagaki, B. Jenichen, C. Herrmann, E. Wiebicke, L. Daweritz, and K. H. Ploog, Phys. Rev. B 73, 125324 (2006).

${ }^{3}$ C. Adriano, C. Giles, O. D. D. Couto, M. J. S. P. Brasil, F. Iikawa, and L. Daweritz, Appl. Phys. Lett. 88, 151906 (2006).

${ }^{4}$ Y. Kato et al., Nature 407, 50 (2004).
${ }^{5}$ D. H. Mosca, F. Vidal, and V. H. Etgens, Phys. Rev. Lett. 101, 125503 (2008).

${ }^{6}$ A. K. Das, C. Pampuch, A. Ney, T. Hesjedal, L. Daweritz, R. Koch, and K. H. Ploog, Phys. Rev. Lett. 91, 087203 (2003).

${ }^{7}$ F. Iikawa, P. V. Santos, M. Kastner, F. Schippan, and L. Daweritz, Phys. Rev. B 65, 205328 (2002).

${ }^{8}$ J. Mohanty, T. Hesjedal, A. Ney, Y. Takagaki, R. Koch, L. Daweritz, and K. H. Ploog, Appl. Phys. Lett. 83, 2829 (2003).

${ }^{9}$ L. N. Coelho, B. R. A. Neves, R. Magalhães-Paniago, F. C. Vicentin, H. Westfahl, R. M. Fernandes, F. Iikawa, L. Daweritz, C. Spezzani, and M. Sacchi, J. Appl. Phys. 100, 083906 (2006).

${ }^{10}$ V. Garcia, H. Jaffres, M. Eddrief, M. Marangolo, V. H. Etgens, and J.-M. George, Phys. Rev. B 72, 081303(R) (2005).

${ }^{11}$ R. H. Wilson and J. S. Kasper, Acta Crystallogr. 17, 95 (1964).

${ }^{12}$ B. T. M. Willis and H. Rooksby, Proc. Phys. Soc., London, Sect. B 67, 290 (1954).

${ }^{13}$ J. B. Goodenough and J. A. Kafalas, Phys. Rev. 157, 389 (1967).

${ }^{14}$ N. Mattoso, M. Eddrief, J. Varalda, A. Ouerghi, D. Demaille, V. H. Etgens, and Y. Garreau, Phys. Rev. B 70, 115324 (2004).

${ }^{15}$ T. Plake, T. Hesjedal, J. Mohanty, M. Kästner, L. Daweritz, and K. H. Ploog, Appl. Phys. Lett. 82, 2308 (2003).

${ }^{16}$ V. M. Kaganer, B. Jenichen, F. Schippan, W. Braun, L. Daweritz, and K. H. Ploog, Phys. Rev. Lett. 85, 341 (2000).

${ }^{17}$ L. B. Steren, J. Milano, V. Garcia, M. Marangolo, M. Eddrief, and V. H. Etgens, Phys. Rev. B 74, 144402 (2006).

${ }^{18}$ L. Daweritz, Rep. Prog. Phys. 69, 2581 (2006).

${ }^{19}$ L. Däweritz, F. Schippan, A. Trampert, M. Kästner, G. Behme, Z. M. Wang, M. Moreno, P. Schützendübe, and K. H. Ploog, J. Cryst. Growth 227-228, 834-838 (2001).

${ }^{20}$ M. Kästner, C. Herrmann, L. Däweritz, and K. H. Ploog, J. Appl. Phys. 92, 5711 (2002).

${ }^{21}$ F. Schippan, G. Behme, L. Daweritz, K. H. Ploog, B. Dennis, K.-U. Neumann, and K. R. A. Ziebeck, J. Appl. Phys. 88, 2766 (2000).

${ }^{22}$ L. Daweritz, L. Wan, B. Jenichen, C. Herrmann, J. Mohanty, A. Trampert, and K. H. Ploog, J. Appl. Phys. 96, 5056 (2004).

${ }^{23}$ T. Plake, M. Ramsteiner, V. M. Kaganer, B. Jnichen, M. Kästner, L. Daweritz, and K. H. Ploog, Appl. Phys. Lett. 80, 2523 (2002).

${ }^{24}$ M. Tortarolo, M. Sirena, J. Milano, L. B. Steren, F. Vidal, B. Rache Salles, V. H. Etgens, M. Eddrief, G. Faini, and L. I. Pietrasanta, Phys. Rev. B. 81, 224406 (2010).

${ }^{25}$ R. Dingreville, J. Qu, and M. Cherkaoui, J. Mech. Phys. Solids 53, 1827 (2005).

${ }^{26}$ Y.-H. Yoo, W. Lee, and H. Shin, Semicond. Sci. Technol. 19, 93 (2004).

${ }^{27}$ R. Engel-Herbert and T. Hesjedal, Phys. Rev. B 78, 235309 (2008).

${ }^{28}$ R. Engel-Herbert, T. Hesjedal, and D. M. Schaadt, Phys. Rev. B. 75, 094430 (2007).

${ }^{29}$ A. Aharoni, J. Appl. Phys. 83, 3432 (1998)

${ }^{30}$ B. D. Cullity and C. D. Graham, Introduction to Magnetic Materials, 2nd ed. (John Wiley \& Sons, 2009). 